\title{
Difference Analysis Of Related Factors In Macrovascular And Microvascular Complications In Chinese Patients With Type 2 Diabetes Mellitus: A Case-Control Study Protocol
}

This article was published in the following Dove Press journal: Diabetes, Metabolic Syndrome and Obesity: Targets and Therapy

\author{
Hang Zhao' \\ Linyi Shu ${ }^{2}$ \\ Wenli Huang ${ }^{2}$ \\ Wenzhao Wang ${ }^{2}$ \\ Guangyao Song' \\ 'Endocrinology Department, Hebei \\ General Hospital, Shijiazhuang, Hebei \\ 05005I, People's Republic of China; \\ ${ }^{2}$ Department of Internal Medicine, Hebei \\ Medical University, Shijiazhuang, Hebei \\ 0500 I7, People's Republic of China
}

\begin{abstract}
Introduction: Despite the increasingly young age at diabetes onset and the increasing number of deaths caused by type 2 diabetes mellitus (T2DM), why some patients develop macrovascular complications but others develop microvascular complications remains controversial and unclear. Notably, some patients have good glucose control but still develop vascular complications, whereas some patients have retinopathy with neither nephropathy nor neuropathy. This study will be performed to explore the risk factors for T2DM complications in Chinese patients.

Methods and analysis: Patients with T2DM and healthy people will be recruited from Hebei General Hospital from September 2019 to September 2020. The subjects will be grouped into a control group, T2DM without vascular complications group, T2DM with macrovascular complications group, and T2DM with microvascular complications group; they will then be further subgrouped. The following data will be collected after admission: demographic information such as age, sex, and education; relevant medical history such as duration of diabetes, family history of first-degree relatives with diabetes, and age at diagnosis of diabetes; and anthropometric and blood indicators such as weight, waist circumference, fasting blood glucose level, C-peptide level, total cholesterol level, and triglyceride level. The statistical analysis will be performed using SPSS 22.0 (IBM Corp., Armonk, NY, USA). A $P$ value of $<0.05$ will be considered statistically significant. The $\chi^{2}$ test, one-way analysis of variance and the rank sum test will be used to analyze differences between the groups. Logistic regression will be used to analyze the risk factors for macrovascular and microvascular complications of T2DM.
\end{abstract}

Keywords: diabetes mellitus, type 2, diabetic angiopathies, risk factors

\section{Introduction}

Type 2 diabetes mellitus (T2DM) has become a global epidemic disease. In 2017, an estimated 451 million patients aged 18 to 99 years had T2DM worldwide, and this number of patients is predicted to increase to 693 million by $2045^{1}$. The global health-care expenditure for patients with T2DM is currently estimated at $\$ 85$ billion. ${ }^{1}$ In 2013, monitoring of chronic diseases and risk factors in China showed that the prevalence of T2DM in people older than 18 was $10.4 \%{ }^{2}$

T2DM is a common metabolic disorder that is associated with chronic complications such as macrovascular and microvascular disease. Almost half of the general population (49.7\%) has T2DM but has not been diagnosed. ${ }^{1}$ The onset of T2DM is
Correspondence: Guangyao Song Endocrinology Department, Hebei General Hospital, 348 Heping West Road, Shijiazhuang, Hebei 05005I, People's

Republic of China

Tel +86 3II 85988556

Email guangyaosong123@163.com 
insidious and often diagnosed approximately 4 to 7 years after the onset of illness. ${ }^{3}$ In patiens with T2DM, the patients often develop associated complications. Arterial stenosis results in reduced blood flow to the heart, brain, or limbs, leading to a variety of macrovascular complications. ${ }^{4} \mathrm{~T} 2 \mathrm{DM}$ can also damage microvessels, as patients who develop diabetes retinopathy, diabetes nephropathy, and diabetes neuropathy. Failure to prevent and detect diabetes the macrovascular or microvascular complications of T2DM can affect patients' health and quality of life, potentially resulting in visual impairment, pain, paresthesia, muscle weakness, diabetes autonomic dysfunction, blindness, myocardial infarction, cardiac arrest, renal failure, and other serious consequences. ${ }^{5} \mathrm{~T} 2 \mathrm{DM}$ has long been recognized as a disease that affects older people, but increasingly more young people aged 20-40 years are being found to have this disease. In 2017, approximately 5 million people aged 20 to 99 years died of T2DM worldwide. ${ }^{1}$ Given the younger age at onset and the increasing number of deaths caused by T2DM, we should pay more attention to its occurrence and development. The pathogenesis of diabetes complications is not fully understood, and why some patients develop macrovascular complications and others develop microvascular complications remains controversial and unclear. ${ }^{6}$ Specifically, why do some patients with good glucose control still develop macrovascular or microvascular complications? Why do some patients develop diabetes retinopathy without diabetes nephropathy or diabetes neuropathy ${ }^{6-8}$ Given the various hazards of the vascular complications of T2DM and many outstanding questions in relation to this disease, we plan to examine subgroups of patients with T2DM who have developed diabetes complications and identify factors that influence disease progression.

Studies have shown that early prevention and identification of risk factors for complications may delay or prevent the further development of T2DM into conditions such as blindness, end-stage renal disease, foot ulcers, heart failure and hemiplegia. Screening for macrovascular and microvascular complications is important for delaying, preventing, and managing T2DM, as well as reducing complications and health care expenditure.

\section{Methods And Analysis}

This will be a case-control study

\section{Aims Of Study}

In summary, this study will be performed to:
(I) determine the proportion of macrovascular and microvascular complications in Chinese patients with T2DM,

(II) determine the differences in the relevant indicators between the subgroups of diabetes vascular complications and

(III) identify the risk factors for the various macrovascular and microvascular complications of T2DM.

Risk factors may include demographic information (age, gender, education, place of residence, occupation, income), relevant medical history (duration of T2DM, family history of first-degree relatives of T2DM, family history of hypertension, age at diagnosis of T2DM, sleep, history of smoking, history of drinking, exercise), anthropometric indicators (height, weight, BMI, waist circumference, hip circumference, blood pressure, heart rate) and blood indicators (fasting blood glucose, C-peptide, total cholesterol, triglyceride, high-density lipoprotein, low-density lipoprotein, apolipoprotein A1, apolipoprotein B, glycated hemoglobin, creatinine, $2 \mathrm{hr}$ postprandial blood glucose, $2 \mathrm{hr}$ postprandial C-peptide, $2 \mathrm{hr}$ postprandial total cholesterol, $2 \mathrm{hr}$ postprandial triglycerides, $2 \mathrm{hr}$ postprandial high-density lipoprotein, $2 \mathrm{hr}$ postprandial low-density lipoprotein).

\section{Subjects}

Patients with T2DM and healthy subjects will be recruited from Hebei General Hospital Medical Examination Center from September 2019 to September 2020. Two researchers (Linyi Shu and Wenli Huang) will explain to each subject the purpose, significance, and process of the study and the tests to be performed. All subjects will participate in the study on a voluntary basis and will be enrolled after providing written informed consent. The investigator will ask patients whether they have been previously diagnosed with macrovascular or microvascular complications of T2DM; if they have a previous diagnosis, the investigator will request medical records. If patients do not have an existing diagnosis or are uncertain, the relevant examinations will be carried out.

\section{Definitions}

Macrovascular complications of T2DM refer to atherosclerosis affecting the aorta, coronary artery, basilar artery, or renal artery. Microvascular complications of T2DM refer to microcirculation abnormalities. Microvascular lesions mainly manifest in the retina, kidney, and nervous tissue. 


\section{Grouping Of Patients}

If a patient has two or more complications at the same time, the patient will be assigned to two or more than groups according to the clinical assessment as follows:

(I) Control group: healthy subjects

(II) T2DM without vascular complications group: patients with T2DM who have not developed vascular complications

(III) T2DM with microvascular complications group: patients with T2DM who have developed microvascular but no macrovascular complications

Subgroups: (i). diabetes retinopathy group, (ii) diabetes nephropathy group, and (iii) diabetes peripheral neuropathy group

(IV) T2DM with macrovascular complications group: patients with who have developed macrovascular but no microvascular complications

Subgroups: (i) cerebrovascular disease group, (ii) coronary heart disease group, and (iii) peripheral vascular disease group

\section{Selection Criteria Inclusion Criteria}

(I) Diagnostic criteria for T2DM

China currently uses the diagnostic criteria for T2DM issued by the World Health Organization Diabetes Expert Committee in 1999, shown in Table 1.

(I) Diagnostic criteria for macrovascular complications.

i. Cerebrovascular disease: computed tomography or magnetic resonance imaging findings will be used as the diagnostic criteria.

ii. Coronary heart disease: typical angina or myocardial infarction symptoms, confirmed by electrocardiography, plate test, or coronary angiography.

iii. Peripheral vascular disease: typical symptoms such as intermittent claudication, confirmed by ultrasound, angiography, or other tests as indicated.

(II) Diagnostic criteria for microvascular complications.

i. Diabetes retinopathy: confirmed by an ophthalmologist using ophthalmoscopy or fundus fluorescein angiography.
Table I Diagnostic Criteria For T2DM

\begin{tabular}{|l|l|}
\hline Diagnostic Criteria & $\begin{array}{l}\text { Venous Plasma Glucose } \\
\text { (mmol/L) }\end{array}$ \\
\hline $\begin{array}{l}\text { Diabetes symptoms + random } \\
\text { blood glucose }\end{array}$ & $\geq 11.1$ \\
\hline Or fasting blood glucose (FPG) & $\geq 7.0$ \\
\hline OGTT 2 hrs blood glucose & $\geq 11.1$ \\
\hline
\end{tabular}

Notes: Symptoms of diabetes: polyuria, polydipsia, and unexplained weight loss. It needs to be tested again to confirm, so the diagnosis can be established. Random blood glucose refers to blood glucose at any time of the day regardless of the meal time.

Abbreviations: OGTT, Oral glucose tolerance test; T2DM, type 2 diabetes mellitus.

ii. Diabetes nephropathy: at present, there are no unified clinical diagnostic criteria for diabetes nephropathy in China. The consensus on prevention and treatment of diabetes nephropathy (2014 edition) suggests that among most T2DM patients, one of the following can be used to diagnose diabetes nephropathy: heavy albuminuria ( $>0.3 \mathrm{~g} / 24 \mathrm{~h}$ ); diabetes retinopathy with any stage of chronic kidney disease. Nondiabetes nephropathy should be excluded during diagnosis. Renal puncture pathology should be used for identification when it is difficult to identify.

iii. Diabetes peripheral neuropathy: (a) a clear history of diabetes; (b) neuropathy that occurs at or after the diagnosis of T2DM; (c) clinical symptoms and signs consistent with of diabetes peripheral neuropathy; (d) clinical symptoms (such as pain, numbness, or paresthesia) and one abnormality among the following five examinations: sputum reflex, acupuncture pain sense, vibration sense, pressure sense, and temperature sense) (or two abnormalities among the of five examinations in patients without clinical symptoms); and (e) exclusion of neuropathy of other causes.

\section{Exclusion Criteria}

(i) Failure to meet the above diagnostic criteria (ii) Infection at the time of admission, such as pneumonia or urinary tract infection

(ii) Pregnancy or lactation

(iii) Mental illness caused by physical illness or psychoactive substances 
(iv) Incomplete information

(v) Acute phase of diseases such as cerebral infarction, myocardial infarction, or malignant tumor

(vi) Unsuitability for the study

\section{Data Collection}

On the day of admission, a researcher will collect demographic information in the form of questions and answers: education (primary school, junior high school, high school, or university and above), place of residence (rural or urban), occupation (mental worker or manual worker), income $(<2000,2000-5000$, or $>5000$ yuan), age, and sex.

Unified measurement of the body indicators will be performed on the day of admission: height, weight, waist circumference, hip circumference, blood pressure, and heart rate.

The relevant medical history will include information regarding the disease course, family history of first-degree relatives with diabetes, family history of hypertension, age at diagnosis of diabetes, sleep (good, average or poor), history of smoking, history of drinking, exercise [rarely, occasionally (1-2 times a week), or often ( $>3$ weeks per week)], other diseases, and medications.

For the oral glucose tolerance test, the participants will be given a normal diet the day before the test and then fasted after 22:00. The next morning, the fasting blood glucose, C-peptide, cholesterol, triglyceride, high-density lipoprotein, low-density lipoprotein, apolipoprotein A1, apolipoprotein B, glycated hemoglobin, and creatinine levels will be measured. After collecting the fasting blood samples, $75 \mathrm{~g}$ of anhydrous glucose will be dissolved in $300 \mathrm{~mL}$ of water, which the patient will drink within 5 mins. The time point at which the first mouthful is taken will be recorded; 2 hrs later, the blood glucose, Cpeptide, total cholesterol, triglyceride, high-density lipoprotein and low-density lipoprotein levels will be measured.

\section{Planned Analyses}

The statistical analysis will be performed using SPSS 22.0 (IBM Corp., Armonk, NY, USA). Numerical variables with a normal distribution will be expressed as mean \pm standard deviation, and those with a non-normal distribution will be expressed as median (interquartile range). A $P$ value of $<0.05$ will be considered statistically significant. Categorical data will be analyzed using the $\chi^{2}$ test, whereas data with a normal distribution will be analyzed using oneway analysis of variance. If a normal distribution is not met, the rank sum test will be used to analyze differences between the groups. Logistic regression will be used to analyze the risk factors for macrovascular and microvascular complications in patients with T2DM. The final information is shown in Table 2-4. A flow chart of the study is shown in Figure 1.

\section{Ethics And Dissemination}

This study has been approved by the Ethics Committee of Hebei General Hospital and will be carried out according to the guidelines of the Declaration of Helsinki.

\section{Patient Rights}

Patiens can withdraw at any time. After all indicators and test results are available, we will explain the state of the illness to the patient. The paper version of the report will be issued to the patient within one week after admission. We will bear the extra cost of the patients.

\section{Discussion}

The data obtained from this study will allow calculation of the proportions of various macrovascular and microvascular complications in patients with T2DM. The United Kingdom Prospective Diabetes Study reported that the current incidence of diabetes macrovascular complications is $22.9 \%$ and that the incidences of cerebrovascular disease, peripheral vascular disease, and myocardial infarction are $3.7 \%, 1.0 \%$, and $15.3 \%$, respectively. ${ }^{9}$ Traditional risk factors such as hypertension, obesity, and hypercholesterolemia are associated with cardiovascular mortality. One study showed that the incidences of diabetes retinopathy, diabetes nephropathy, and diabetes neuropathy were: $14.8 \%, 15.0 \%, 5.6 \%$, respectively, in patients with T2DM.

The mechanisms of these chronic vascular complications are not fully understood, but it is generally believed that chronic hyperglycemia, elevated levels of reactive oxygen species, decreased levels of nitric oxide, and accumulation of advanced glycation end products (AGEs) lead to changes in vascular responses. ${ }^{10}$ Extensive deposition of AGEs inside and outside cells exacerbates persistent vascular injury induced by hyperglycemia. AGEs increase vascular permeability, induce procoagulant activity, cause intimal migration of $\mathrm{T}$ cells and macrophages, and impair endothelium-dependent relaxation. ${ }^{11}$ Inhibition of AGEs attenuates the severity of atherosclerosis associated with T2DM. Hyperlipidemia causes endothelial dysfunction, which leads to the formation of diabetes retinopathy and 
Table 2 Demographic Information In Each Group

\begin{tabular}{|c|c|c|c|c|c|c|c|c|}
\hline & \multirow[t]{2}{*}{ NC } & \multirow[t]{2}{*}{ ND } & \multicolumn{3}{|l|}{ MA } & \multicolumn{3}{|l|}{ MI } \\
\hline & & & CV & CHD & PVD & DR & DN & DPN \\
\hline Age (year, mean $\pm S D)$ & & & & & & & & \\
\hline $\begin{array}{l}\text { Gender } \\
\text { Male (n) } \\
\text { Female (n) }\end{array}$ & & & & & & & & \\
\hline $\begin{array}{l}\text { Education } \\
\text { Primary school (n) } \\
\text { Junior high school (n) } \\
\text { High school (n) } \\
\geqq \text { University (n) }\end{array}$ & & & & & & & & \\
\hline $\begin{array}{c}\text { Place of residence } \\
\text { Rural area (n) } \\
\text { Urban area (n) }\end{array}$ & & & & & & & & \\
\hline $\begin{array}{l}\text { Occupation } \\
\text { Mental worker (n) } \\
\text { Manual worker (n) }\end{array}$ & & & & & & & & \\
\hline $\begin{array}{l}\text { Income } \\
\qquad \begin{array}{l}\text { RMB }<2000(n) \\
\text { RMB } 2000-5000(n) \\
\text { RMB }>5000 \text { (n) }\end{array}\end{array}$ & & & & & & & & \\
\hline
\end{tabular}

Abbreviations: NC, Control group; ND, T2DM without vascular complications group; MA, T2DM with macrovascular complications group; CV, Cerebrovascular disease group; CHD, Coronary heart disease group; CV, Peripheral vascular disease group; MI, T2DM with microvascular complications group; DR, Diabetes retinopathy group; DN, Diabetes nephropathy group; DPN, Diabetes peripheral neuropathy group; T2DM, type 2 diabetes mellitus.

Table 3 Relevant Medical History In Each Group

\begin{tabular}{|c|c|c|c|c|c|c|c|c|}
\hline & \multirow[t]{2}{*}{ NC } & \multirow[t]{2}{*}{ ND } & \multicolumn{3}{|l|}{ MA } & \multicolumn{3}{|l|}{ MI } \\
\hline & & & CV & CHD & PVD & DR & DN & DPN \\
\hline $\begin{array}{l}\text { Duration of T2DM (year, mean } \pm S[ \\
\text { Family history of first-degree relati } \\
\text { Family history of hypertension ( } \mathrm{n} \text { ) } \\
\text { Age at diagnosis of T2DM (year) }\end{array}$ & & & & & & & & \\
\hline $\begin{array}{l}\text { Sleep }(n) \\
\text { Good } \\
\text { Average } \\
\text { Poor }\end{array}$ & & & & & & & & \\
\hline $\begin{array}{l}\text { History of smoking }(n) \\
\text { History of drinking }(n)\end{array}$ & & & & & & & & \\
\hline $\begin{array}{l}\text { Exercise }(n) \\
\text { Rarely: }<1 \text { time } \\
\text { Occasionally: I-2 times a week } \\
\text { Often: }>3 \text { weeks a week }\end{array}$ & & & & & & & & \\
\hline
\end{tabular}

Abbreviations: NC, Control group; ND, T2DM without vascular complications group; MA, T2DM with macrovascular complications group; CV, Cerebrovascular disease group; CHD, Coronary heart disease group; CV, Peripheral vascular disease group; MI, T2DM with microvascular complications group; DR, Diabetes retinopathy group; DN, Diabetes nephropathy group; DPN, Diabetes peripheral neuropathy group; T2DM, type 2 diabetes mellitus. 
Table 4 Anthropometric Indicators And Blood Indicators In Each Group (meantsd)

\begin{tabular}{|c|c|c|c|c|c|c|c|c|}
\hline & \multirow[t]{2}{*}{ NC } & \multirow[t]{2}{*}{ ND } & \multicolumn{3}{|l|}{ MA } & \multicolumn{3}{|l|}{ MI } \\
\hline & & & CV & CHD & PVD & DR & DN & DPN \\
\hline 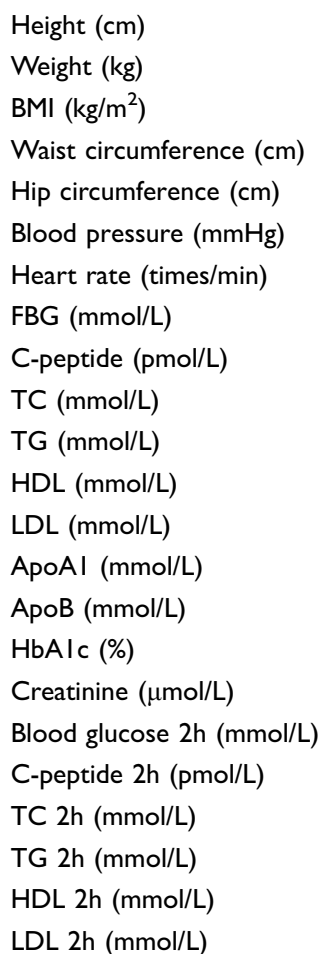 & & & & & & & & \\
\hline
\end{tabular}

Abbreviations: NC, Control group; ND, T2DM without vascular complications group; MA, T2DM with macrovascular complications group; CV, Cerebrovascular disease group; CHD, Coronary heart disease group; CV, Peripheral vascular disease group; MI, T2DM with microvascular complications group; DR, Diabetes retinopathy group; DN, Diabetes nephropathy group; DPN, Diabetes peripheral neuropathy group; BMI, body mass index; FBG, fasting blood glucose; TC, total cholesterol; TG, triglyceride; HDL, high-density lipoprotein; LDL, low-density lipoprotein; HbAIc, glycated hemoglobin; T2DM, type 2 diabetes mellitus.

macular edema by reducing the bioavailability of nitric oxide and destroying the blood-retinal barrier, causing leakage of serum lipids and lipoproteins. ${ }^{12}$ However, Cetin et $\mathrm{al}^{13}$ found no a correlation between blood lipid levels and the severity of diabetes retinopathy. In addition, the effects of statins on microvascular complications in patients with T2DM are controversial. One study in Denmark showed that the use of statins before the diagnosis of T2DM could reduce the incidenct of diabetes retinopathy and neuropathy, ${ }^{14}$ while Mansi et al ${ }^{15}$ reported that statins were associated with an increased risk of complications of T2DM.

Inflammation is closely associated with atherosclerosis ${ }^{16}$ and T2DM. ${ }^{17}$ Dasu et al $^{18}$ reported that monocytes in highconcentration glucose in patients with poorly controlled T2DM are activated that and inflammatory mediators such as protein kinase $\mathrm{C}$ and nuclear factor- $\mathrm{\kappa B}$ are also activated. In vitro studies have reported similar effects of hyperglycemia on the atherogenic effects of $\mathrm{T}$ lymphocytes and inflammatory cells. ${ }^{19}$
Previous studies have concluded that the age of the patient and the duration of T2DM are the main predictors of complications. ${ }^{20}$ Patients with a $>20$-year history of T2DM have a 30\% higher risk of complications than patients with a $<10$-year history of T2DM. For each additional year of age, the incidence of microvascular complications increases by $4 \%$ and that of macrovascular complications increases by $2 \%$. Patients with T2DM who have poor glucose control and a longer duration of diabetes have a higher prevalence of large blood vessels and microvessels. ${ }^{4}$ A study of 400 patients with T2DM aged 30-60 years in a hospital in Bangladesh showed that female sex, a housewife status, a low level physical activity, increased age, poor glucose control, hypertension, and a longer duration of diabetes were likely to be risk factors for microvascular disease. ${ }^{21}$ To date, studies have shown that the serum asymmetric dimethylarginine and arginine levels can predict macrovascular and microvascular complications in patients with T2DM. ${ }^{22}$ 


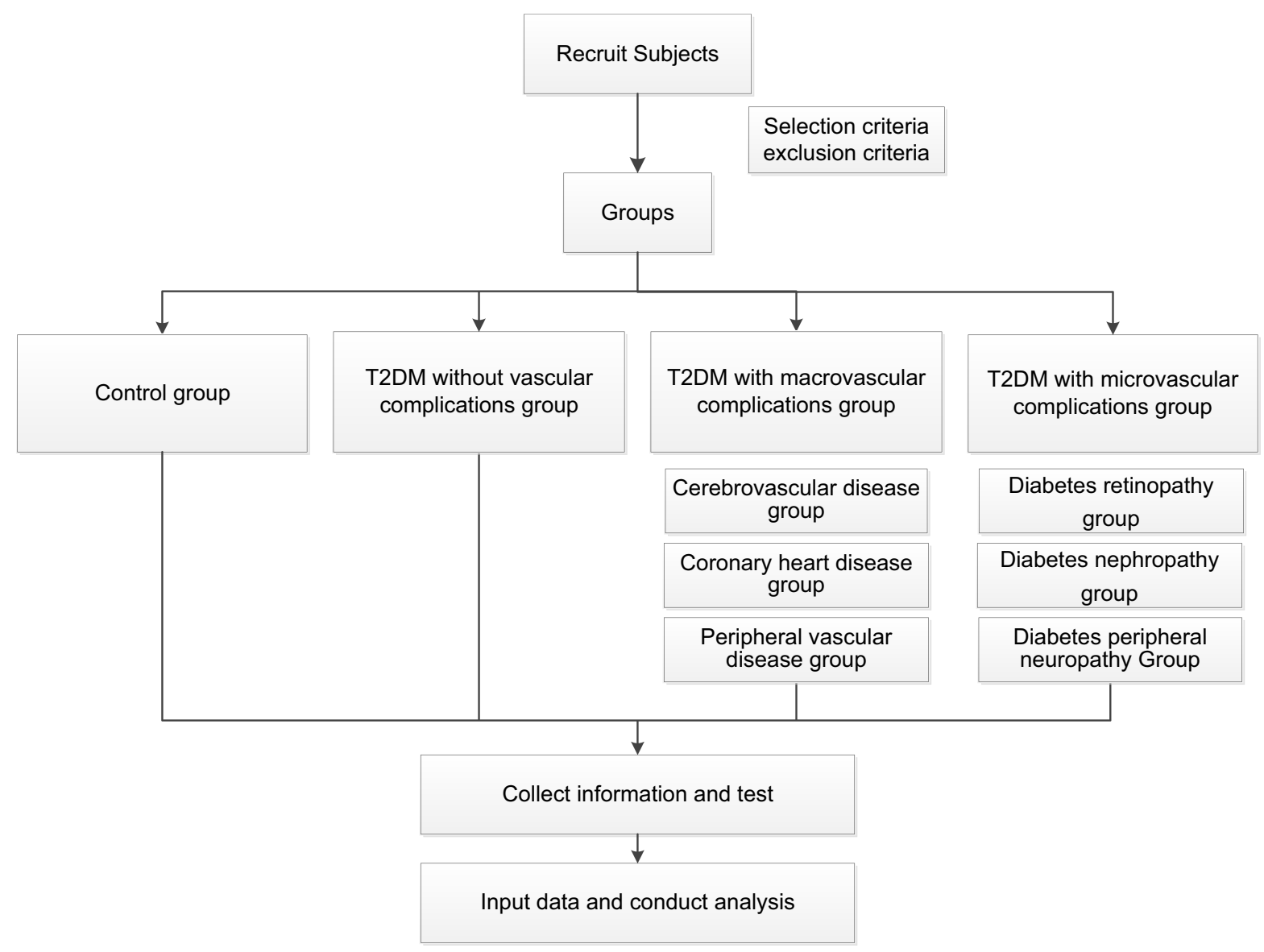

Figure I Flow chart of the study from recruiting subjects to conducting analysis.

Previous studies of T2DM complications have been be limited to large blood vessels or microvessels. Our study combines the observation of macrovascular disease and microvascular disease and will use stratified analysis of the influencing factors in each subgroup (macrovascular diseases include cardiovascular disease, cerebrovascular disease, and peripheral vascular disease; microvascular diseases include diabetes retinopathy, diabetes nephropathy, and diabetes neuropathy).

Our study can explain why patients with T2DM develop different complications. If differential factors are found, it can guide clinical work in this field. Targeted positive preventions and interventions could be conducted to reduce the incidence of complications in patients with different risk factors.

\section{Limitations}

Case studies are more limited and less representative than multi-center, large-sample studies. The enrollment of all patients with T2DM from one hospital will lead to a bias in the reported results.

\section{Acknowledgments}

We thank Catherine Deeprose, $\mathrm{PhD}$ and Angela Morben, DVM, ELS, from Liwen Bianji, Edanz Editing China, for editing the English text of a draft of this manuscript. We also thank the patient advisers in the study. This study is being supported by a grant from the Natural Science Foundation of Hebei Province (No. H2018307071).

\section{Disclosure}

The authors report no conflicts of interest in this work.

\section{References}

1. Cho NH, Shaw JE, Karuranga S, et al. IDF Diabetes Atlas: global estimates of diabetes prevalence for 2017 and projections for 2045. Diabetes Res Clin Pract. 2018;138:271-281. doi:10.1016/j.diabres. 2018.02.023

2. Wang L, Gao P, Zhang M, et al. Prevalence and ethnic pattern of diabetes and prediabetes in China in 2013. JAMA. 2017;317(24):25152523. doi:10.1001/jama.2017.7596

3. Harris MI, Klein R, Welborn TA, Knuiman MW. Onset of NIDDM occurs at least 4-7 yr before clinical diagnosis. Diabetes Care. 1992;15(7):815-819. doi:10.2337/diacare.15.7.815 
4. Alaboud AF, Tourkmani AM, Alharbi TJ, et al. Microvascular and macrovascular complications of type 2 diabetic mellitus in Central, Kingdom of Saudi Arabia. Saudi Med J. 2016;37(12):1408-1411. doi:10.15537/smj.2016.12.17062

5. Sina M, Graffy J, Simmons D. Associations between barriers to selfcare and diabetes complications among patients with type 2 diabetes. Diabetes Res Clin Pract. 2018;141:126-131. doi:10.1016/j.diabres. 2018.04.031

6. Mohammedi K, Woodward M, Marre M, et al. Comparative effects of microvascular and macrovascular disease on the risk of major outcomes in patients with type 2 diabetes. Cardiovasc Diabetol. 2017;16(1):95. doi:10.1186/s12933-017-0624-5

7. Karabag T, Kaya A, Temizhan A, et al. The influence of homocysteine levels on endothelial function and their relation with microvascular complications in T2DM patients without macrovascular disease. Acta Diabetol. 2007;44(2):69-75. doi:10.1007/s00592-007-0245-7

8. Ozmen B, Boyvada S. The relationship between self-monitoring of blood glucose control and glycosylated haemoglobin in patients with type 2 diabetes with and without diabetic retinopathy. J Diabetes Complications. 2003;17(3):128-134.

9. Stratton IM, Adler AI, Neil HA, et al. Association of glycaemia with macrovascular and microvascular complications of type 2 diabetes (UKPDS 35): prospective observational study. BMJ. 2000;321 (7258):405-412. doi:10.1136/bmj.321.7258.405

10. Lee MY, Hsiao PJ, Huang JC, Hsu WH, Chen SC, Shin SJ. Association between metabolic syndrome and micro- and macrovascular disease in type 2 diabetic mellitus. Am J Med Sci. 2018;355 (4):342-349. doi:10.1016/j.amjms.2017.12.004

11. Lu M, Luo Y, Hu P, Dou L, Huang S. Tanshinone IIA inhibits AGEsinduced proliferation and migration of cultured vascular smooth muscle cells by suppressing ERK1/2 MAPK signaling. Iran J Basic Med Sci. 2018;21(1):83-88. doi:10.22038/IJBMS.2017.20100.5276

12. Golubovic-Arsovska M. Association of dyslipidaemia with macular oedema and hard exudates in diabetic maculopathy. Prilozi. 2007;28 (2):149-160.

13. Cetin EN, Bulgu Y, Ozdemir S, Topsakal S, Aybek H. Association of serum lipid levels with diabetic retinopathy. Int J Ophthalmol. 2013;6 (3):346-349. doi:10.3980/j.issn.2222-3959.2013.03.17
14. Nielsen SF, Nordestgaard BG. Statin use before diabetes diagnosis and risk of microvascular disease: a nationwide nested matched study. Lancet Diabetes Endocrinol. 2014;2(11):894-900. doi:10. 1016/S2213-8587(14)70173-1

15. Mansi I, Frei CR, Wang CP, Mortensen EM. Statins and new-onset diabetes mellitus and diabetic complications: a retrospective cohort study of US healthy adults. J Gen Intern Med. 2015;30(11):15991610. doi:10.1007/s11606-015-3335-1

16. Jialal I, Vikram NK. Inflammation and atherosclerosis: fulfilling Koch's postulates. Ther Adv Cardiovasc Dis. 2018;12(1):5-6. doi: $10.1177 / 1753944717744740$

17. Pouvreau C, Dayre A, Butkowski EG, Jong BD, Jelinek HF. Inflammation and oxidative stress markers in diabetes and hypertension. J Inflamm Res. 2018;11:61-68. doi:10.2147/JIR.S148911

18. Dasu MR, Devaraj S, Jialal I. High glucose induces IL-1beta expression in human monocytes: mechanistic insights. Am J Physiol Endocrinol Metab. 2007;293(1):E337-E346. doi:10.1152/ajpendo.00 718.2006

19. Venugopal SK, Devaraj S, Yang T, Jialal I. Alpha-tocopherol decreases superoxide anion release in human monocytes under hyperglycemic conditions via inhibition of protein kinase $\mathrm{C}$-alpha. Diabetes. 2002;51(10):3049-3054. doi:10.2337/diabetes.51.10.3049

20. Zoungas S, Woodward M, Li Q, et al. Impact of age, age at diagnosis and duration of diabetes on the risk of macrovascular and microvascular complications and death in type 2 diabetes. Diabetologia. 2014;57(12):2465-2474. doi:10.1007/s00125-014-3369-7

21. Khanam PA, Hoque S, Begum T, Habib SH, Latif ZA. Microvascular complications and their associated risk factors in type 2 diabetes mellitus. Diabetes Metab Syndr. 2017;2:S577-S581. doi:10.1016/j. dsx.2017.04.007

22. Ganz T, Wainstein J, Gilad S, Limor R, Boaz M, Stern N. Serum asymmetric dimethylarginine and arginine levels predict microvascular and macrovascular complications in type 2 diabetes mellitus. Diabetes Metab Res Rev. 2017;33:e2836. doi:10.1002/dmrr.v33.2

\section{Publish your work in this journal}

Diabetes, Metabolic Syndrome and Obesity: Targets and Therapy is an international, peer-reviewed open-access journal committed to the rapid publication of the latest laboratory and clinical findings in the fields of diabetes, metabolic syndrome and obesity research. Original research, review, case reports, hypothesis formation, expert opinion and commentaries are all considered for publication. The manuscript management system is completely online and includes a very quick and fair peer-review system, which is all easy to use. Visit http://www.dovepress.com/testimonials.php to read real quotes from published authors.

Submit your manuscript here: https://www.dovepress.com/diabetes-metabolic-syndrome-and-obesity-targets-and-therapy-journal 